\title{
Study of Beauty Hadron Decays into Pairs of Charm Hadrons
}

\author{
R. Aaij et al. \\ (LHCb Collaboration)
}

(Received 17 March 2014; published 21 May 2014)

First observations of the decays $\Lambda_{b}^{0} \rightarrow \Lambda_{c}^{+} D_{(s)}^{-}$are reported using data corresponding to an integrated luminosity of $3 \mathrm{fb}^{-1}$ collected at 7 and $8 \mathrm{TeV}$ center-of-mass energies in proton-proton collisions with the LHCb detector. In addition, the most precise measurement of the branching fraction $\mathcal{B}\left(B_{s}^{0} \rightarrow D^{+} D_{s}^{-}\right)$is made and a search is performed for the decays $B_{(s)}^{0} \rightarrow \Lambda_{c}^{+} \Lambda_{c}^{-}$. The results obtained are

$$
\begin{aligned}
\mathcal{B}\left(\Lambda_{b}^{0} \rightarrow \Lambda_{c}^{+} D^{-}\right) / \mathcal{B}\left(\Lambda_{b}^{0} \rightarrow \Lambda_{c}^{+} D_{s}^{-}\right) & =0.042 \pm 0.003 \text { (stat) } \pm 0.003 \text { (syst) }, \\
{\left[\frac{\mathcal{B}\left(\Lambda_{b}^{0} \rightarrow \Lambda_{c}^{+} D_{s}^{-}\right)}{\mathcal{B}\left(\bar{B}^{0} \rightarrow D^{+} D_{s}^{-}\right)}\right] /\left[\frac{\mathcal{B}\left(\Lambda_{b}^{0} \rightarrow \Lambda_{c}^{+} \pi^{-}\right)}{\mathcal{B}\left(\bar{B}^{0} \rightarrow D^{+} \pi^{-}\right)}\right] } & =0.96 \pm 0.02 \text { (stat) } \pm 0.06 \text { (syst) } \\
\mathcal{B}\left(B_{s}^{0} \rightarrow D^{+} D_{s}^{-}\right) / \mathcal{B}\left(\bar{B}^{0} \rightarrow D^{+} D_{s}^{-}\right) & =0.038 \pm 0.004 \text { (stat) } \pm 0.003 \text { (syst), } \\
\mathcal{B}\left(\bar{B}^{0} \rightarrow \Lambda_{c}^{+} \Lambda_{c}^{-}\right) / \mathcal{B}\left(\bar{B}^{0} \rightarrow D^{+} D_{s}^{-}\right) & <0.0022[95 \% \text { C.L.] } \\
\mathcal{B}\left(B_{s}^{0} \rightarrow \Lambda_{c}^{+} \Lambda_{c}^{-}\right) / \mathcal{B}\left(B_{s}^{0} \rightarrow D^{+} D_{s}^{-}\right) & <0.30[95 \% \text { C.L.]. }
\end{aligned}
$$

Measurement of the mass of the $\Lambda_{b}^{0}$ baryon relative to the $\bar{B}^{0}$ meson gives $M\left(\Lambda_{b}^{0}\right)-M\left(\bar{B}^{0}\right)=339.72 \pm 0.24$ (stat) \pm 0.18 (syst) $\mathrm{MeV} / c^{2}$. This result provides the most precise measurement of the mass of the $\Lambda_{b}^{0}$ baryon to date.

DOI: 10.1103/PhysRevLett.112.202001

PACS numbers: 14.20.Mr, 13.30.-a

Hadrons are systems of quarks bound by the strong interaction, described at the fundamental level by quantum chromodynamics (QCD). Low-energy phenomena, such as the binding of quarks and gluons within hadrons, lie in the nonperturbative regime of QCD and are difficult to calculate. Much progress has been made in recent years in the study of beauty mesons [1]; however, many aspects of beauty baryons are still largely unknown. Many decays of beauty mesons into pairs of charm hadrons have branching fractions at the percent level [2]. Decays of beauty baryons into pairs of charm hadrons are expected to be of comparable size, yet none have been observed to date. If such decays do have sizable branching fractions, they could be used to study beauty-baryon properties. For example, a comparison of beauty meson and baryon branching fractions can be used to test factorization in these decays [3].

Many models and techniques have been developed that attempt to reproduce the spectrum of the measured hadron masses, such as constituent-quark models or lattice QCD calculations [4]. Precise measurements of ground-state beauty-baryon masses are required to permit precision tests of a variety of QCD models [5-11]. The $\Lambda_{b}^{0}$ baryon mass is particularly interesting in this context, since several

* Full author list given at the end of the article.

Published by the American Physical Society under the terms of the Creative Commons Attribution 3.0 License. Further distribution of this work must maintain attribution to the author(s) and the published articles title, journal citation, and DOI. ground-state beauty-baryon masses are measured relative to that of the $\Lambda_{b}^{0}$ [12].

This Letter reports the first observation of the decays $\Lambda_{b}^{0} \rightarrow \Lambda_{c}^{+} D_{s}^{-}$and $\Lambda_{b}^{0} \rightarrow \Lambda_{c}^{+} D^{-}$. The decay $\Lambda_{b}^{0} \rightarrow \Lambda_{c}^{+} D_{s}^{-}$ is used to make the most precise measurement to date of the mass of the $\Lambda_{b}^{0}$ baryon. Improved measurements of the branching fraction $\mathcal{B}\left(B_{s}^{0} \rightarrow D^{+} D_{s}^{-}\right)$and stringent upper limits on $\mathcal{B}\left(B_{(s)}^{0} \rightarrow \Lambda_{c}^{+} \Lambda_{c}^{-}\right)$are also reported. Charge conjugated decay modes are implied throughout this Letter. The data used correspond to an integrated luminosity of 1 and $2 \mathrm{fb}^{-1}$ collected at 7 and $8 \mathrm{TeV}$ center-of-mass energies in $p p$ collisions, respectively, with the LHCb detector.

The $\mathrm{LHCb}$ detector is a single-arm forward spectrometer covering the pseudorapidity range $2<\eta<5$, described in detail in Refs. [13-18]. Samples of simulated events are used to determine selection efficiencies, to model candidate distributions, and to investigate possible background contributions. In the simulation, $p p$ collisions are generated using PYTHIA [19] with a specific LHCb configuration [20]. Decays of hadronic particles are described by EvTGEN [21], in which final-state radiation is generated using Рнотоs [22]. The interaction of the generated particles with the detector and its response are implemented using the GEANT4 toolkit [23] as described in Ref. [24].

In this analysis, signal beauty-hadron candidates are formed by combining charm-hadron candidate pairs reconstructed in the following decay modes: $D^{+} \rightarrow K^{-} \pi^{+} \pi^{+}$, $D_{s}^{+} \rightarrow K^{-} K^{+} \pi^{+}$, and $\Lambda_{c}^{+} \rightarrow p K^{-} \pi^{+}$. The measured invariant mass of each charm-hadron candidate, the resolution on 
which is about $6-8 \mathrm{MeV} / c^{2}$, is required to be within $25 \mathrm{MeV} / c^{2}$ of the nominal value [2]. To improve the resolution of the beauty-hadron mass, the decay chain is fit imposing kinematic and vertex constraints [25]; this includes constraining the charm-hadron masses to their nominal values. To suppress contributions from noncharm decays, the reconstructed charm-hadron decay vertex is required to be downstream of, and significantly displaced from, the reconstructed beauty-hadron decay vertex.

A boosted decision tree (BDT) [26] is used to select each type of charm-hadron candidate. These BDTs use five variables for the charm hadron and 23 for each of its decay products. The variables include kinematic quantities, track and vertex qualities, and particle identification (PID) information. The signal samples used to train the BDTs are obtained from large data sets of $\bar{B}^{0} \rightarrow D^{+} \pi^{-}, \bar{B}_{s}^{0} \rightarrow D_{s}^{+} \pi^{-}$, and $\Lambda_{b}^{0} \rightarrow \Lambda_{c}^{+} \pi^{-}$decays that are background subtracted using weights [27] obtained from fits to the beauty-hadron invariant mass distributions. The background data samples are taken from the charm-hadron and high-mass beautyhadron sidebands in the same data sets. To obtain the BDT efficiency in a given signal decay mode, the kinematical properties and correlations between the two charm hadrons are taken from simulation. The BDT response distributions are obtained from independent data samples of the decays used in the BDT training, weighted to match the kinematics of the signal.

Because of the kinematic similarity of the decays $D^{+} \rightarrow K^{-} \pi^{+} \pi^{+}, D_{s}^{+} \rightarrow K^{-} K^{+} \pi^{+}$, and $\Lambda_{c}^{+} \rightarrow p K^{-} \pi^{+}$, cross feed may occur among beauty-hadron decays into pairs of charm hadrons. For example, cross feed between $D^{+}$ and $D_{s}^{+}$mesons occurs when a $K^{-} h^{+} \pi^{+}$candidate is reconstructed in the $D^{+}$mass region under the $h^{+}=\pi^{+}$ hypothesis and in the $D_{s}^{+}$mass region under the $h^{+}=K^{+}$ hypothesis. In such situations, an arbitration is performed: if the ambiguous track $\left(h^{+}\right)$can be associated to an oppositely charged track to form a $\phi(1020) \rightarrow K^{+} K^{-}$ candidate, the kaon hypothesis is taken, resulting in a $D_{s}^{+}$assignment to the charm-hadron candidate; otherwise, stringent PID requirements are applied to $h^{+}$to choose which hypothesis to take. The efficiency of these arbitrations, which is found to be about $90 \%$ per charm hadron, is obtained using simulated signal decays to model the kinematical properties and $D^{*+} \rightarrow D^{0} \pi^{+}$calibration data for the PID efficiencies. The misidentification probability is roughly $1 \%$ per charm hadron.

Signal yields are determined by performing unbinned extended likelihood fits to the beauty-hadron invariantmass spectra observed in the data. The signal distributions are modeled using a so-called Apollonios function, which is the exponential of a hyperbola combined with a powerlaw low-mass tail [28]. The peak position and resolution parameters are allowed to vary while fitting the data, while the low-mass tail parameters are taken from simulation and fixed in the fits.
Four categories of background contributions are considered: partially reconstructed decays of beauty hadrons where at least one final-state particle is not reconstructed; decays into a single charm hadron and three light hadrons; reflections, defined as cases where the cross-feed arbitration fails to remove a misidentified particle; and combinatorial background. The only partially reconstructed decays that contribute in the mass region studied are those where a single pion or photon is not reconstructed; thus, only final states comprised of $D_{(s)}^{*+}$ or $\Sigma_{c}^{+}$and another charm hadron are considered (e.g., $\Lambda_{b}^{0} \rightarrow \Lambda_{c}^{+} D_{s}^{*-}$ ). These background contributions are modeled using kernel probability density functions (PDFs) [29] obtained from simulation; their yields are free to vary in the fits. Single-charm backgrounds are studied using data that are reconstructed outside of a given charm-hadron mass region. These backgrounds are found to be $\mathcal{O}(1 \%)$ of the size of the signal yield for signal decays containing a $D_{s}^{-}$(e.g., $\bar{B}^{0} \rightarrow D^{+} K^{-} K^{+} \pi^{-}$) and are negligible otherwise. The only non-negligible reflection is found to be $\Lambda_{b}^{0} \rightarrow \Lambda_{c}^{+} D_{s}^{-}$decays misidentified as $\Lambda_{c}^{+} D^{-}$candidates. The invariant-mass distribution for this reflection is obtained from simulation, while the normalization is fixed using simulation and the aforementioned PID calibration sample to determine the fraction of $\Lambda_{b}^{0} \rightarrow \Lambda_{c}^{+} D_{s}^{-}$ decays that are not removed by the cross-feed criteria. Reflections of $\bar{B}^{0} \rightarrow D^{+} D_{s}^{-}$decays misidentified as final states containing $\Lambda_{c}^{+}$particles do not have a peaking structure in the beauty-hadron invariant mass and, therefore, are absorbed into the combinatorial backgrounds, which are modeled using exponential distributions.

Figure 1 shows the invariant mass spectra for the $\Lambda_{b}^{0} \rightarrow \Lambda_{c}^{+} D_{s}^{-}$and $\Lambda_{b}^{0} \rightarrow \Lambda_{c}^{+} D^{-}$candidates. The signal yields obtained are $4633 \pm 69$ and $262 \pm 19$ for $\Lambda_{b}^{0} \rightarrow \Lambda_{c}^{+} D_{s}^{-}$and $\Lambda_{b}^{0} \rightarrow \Lambda_{c}^{+} D^{-}$, respectively. This is the first observation of each of these decays. The ratio of branching fractions determined using the nominal $D_{s}^{-}$[2] and $D^{-}$[30] meson branching fractions and the ratio of efficiencies is

$$
\frac{\mathcal{B}\left(\Lambda_{b}^{0} \rightarrow \Lambda_{c}^{+} D^{-}\right)}{\mathcal{B}\left(\Lambda_{b}^{0} \rightarrow \Lambda_{c}^{+} D_{s}^{-}\right)}=0.042 \pm 0.003 \text { (stat) } \pm 0.003 \text { (syst). }
$$

The similarity of the final states and the shared parent particle result in many cancellations of uncertainties in the determination of the ratio of branching fractions. The remaining uncertainties include roughly equivalent contributions from determining the efficiency-corrected yields and from the ratio of charm-hadron branching fractions (see Table I). The dominant contribution to the uncertainty of the fit PDF is due to the low-mass background contributions, which are varied in size and shape to determine the effect on the signal yield. The uncertainty due to signal model is found to be negligible. The efficiencies of the cross feed and BDT criteria are determined in a data-driven manner that produces small uncertainties. The observed ratio is approximately the ratio of the relevant quark-mixing 

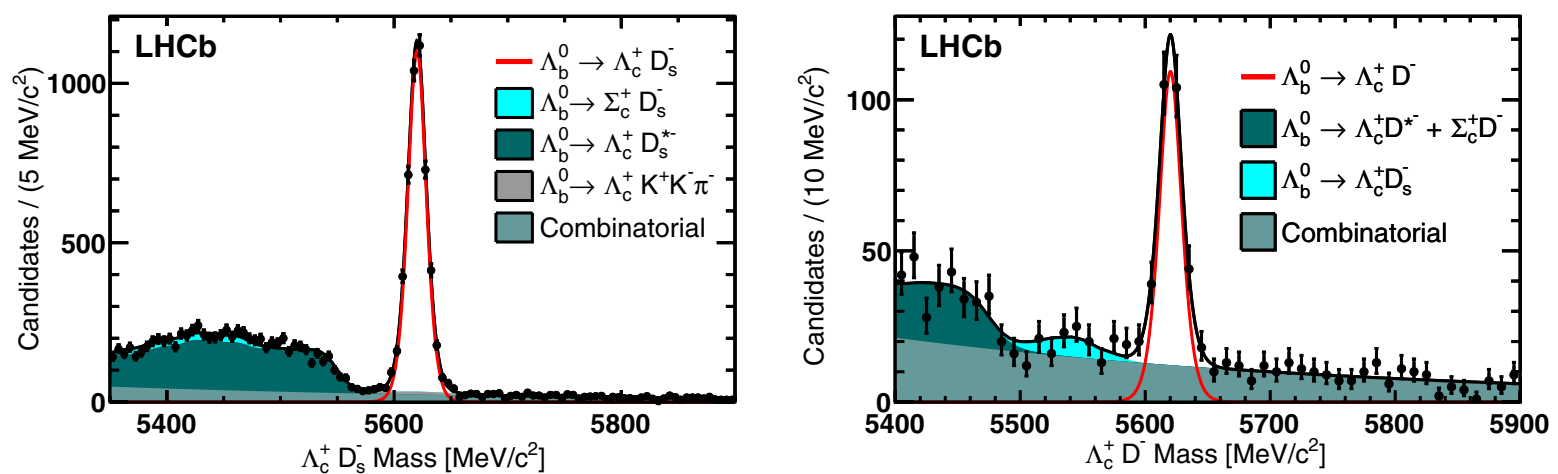

FIG. 1 (color online). Invariant mass distributions for (left) $\Lambda_{b}^{0} \rightarrow \Lambda_{c}^{+} D_{s}^{-}$and (right) $\Lambda_{b}^{0} \rightarrow \Lambda_{c}^{+} D^{-}$candidates with the fits described in the text overlaid.

factors and meson decay constants, $\left|V_{c d} / V_{c s}\right|^{2} \times$ $\left(f_{D} / f_{D_{s}}\right)^{2} \approx 0.034$, as expected assuming nonfactorizable effects are small.

The branching fraction of the decay $\Lambda_{b}^{0} \rightarrow \Lambda_{c}^{+} D_{s}^{-}$is determined relative to that of the $\bar{B}^{0} \rightarrow D^{+} D_{s}^{-}$decay. Using $D^{+} D_{s}^{-}$BDT criteria optimized to maximize the expected $\bar{B}^{0}$ significance, $19395 \pm 145 \bar{B}^{0} \rightarrow D^{+} D_{s}^{-}$decays are observed (see Fig. 2). The measurement of $\mathcal{B}\left(\Lambda_{b}^{0} \rightarrow \Lambda_{c}^{+} D_{s}^{-}\right) /$ $\mathcal{B}\left(\bar{B}^{0} \rightarrow D^{+} D_{s}^{-}\right)$is complicated by the fact that the ratio of the $\Lambda_{b}^{0}$ and $\bar{B}^{0}$ production cross sections, $\sigma\left(\Lambda_{b}^{0}\right) / \sigma\left(\bar{B}^{0}\right)$, depends on the $p_{T}$ of the beauty hadrons [32]. Figure 3 shows the ratio of efficiency-corrected yields, $N\left(\Lambda_{b}^{0} \rightarrow \Lambda_{c}^{+} D_{s}^{-}\right) /$ $N\left(\bar{B}^{0} \rightarrow D^{+} D_{s}^{-}\right)$, as a function of beauty-hadron $p_{T}$. The ratio of branching-fraction ratios is obtained using a fit with the shape of the $p_{T}$ dependence measured in $\mathcal{B}\left(\Lambda_{b}^{0} \rightarrow \Lambda_{c}^{+} \pi^{-}\right) / \mathcal{B}\left(\bar{B}^{0} \rightarrow D^{+} \pi^{-}\right)$[33] and found to be

$$
\begin{gathered}
{\left[\frac{\mathcal{B}\left(\Lambda_{b}^{0} \rightarrow \Lambda_{c}^{+} D_{s}^{-}\right)}{\mathcal{B}\left(\bar{B}^{0} \rightarrow D^{+} D_{s}^{-}\right)}\right] /\left[\frac{\mathcal{B}\left(\Lambda_{b}^{0} \rightarrow \Lambda_{c}^{+} \pi^{-}\right)}{\mathcal{B}\left(\bar{B}^{0} \rightarrow D^{+} \pi^{-}\right)}\right]} \\
=0.96 \pm 0.02 \text { (stat) } \pm 0.06 \text { (syst) } .
\end{gathered}
$$

This result does not depend on the absolute ratio of production cross sections or on any charm-hadron branching fractions. The systematic uncertainties on this result are listed in Table I. The uncertainty in the fit model is due largely to the sizable single-charm background contributions to these modes and to contributions from the fits described in Ref. [33]. The $\mathcal{B}\left(\Lambda_{b}^{0} \rightarrow \Lambda_{c}^{+} \pi^{-}\right) / \mathcal{B}\left(\bar{B}^{0} \rightarrow D^{+} \pi^{-}\right)$result was obtained only using data collected at $\sqrt{s}=7 \mathrm{TeV}$. The ratio $N\left(\Lambda_{b}^{0} \rightarrow \Lambda_{c}^{+} D_{s}^{-}\right) / N\left(\bar{B}^{0} \rightarrow D^{+} D_{s}^{-}\right)$is observed to be consistent in data collected at $\sqrt{s}=7$ and $8 \mathrm{TeV}$. The statistical uncertainty on this comparison is assigned as the systematic uncertainty on the energy dependence of the $\Lambda_{b}^{0}$ and $\bar{B}^{0}$ production fractions. The ratio of branching ratios is consistent with unity, as expected assuming small nonfactorizable effects.

The kinematic similarity of the decay modes $\Lambda_{b}^{0} \rightarrow$ $\Lambda_{c}^{+} D_{s}^{-}$and $\bar{B}^{0} \rightarrow D^{+} D_{s}^{-}$permits a precision measurement of the mass difference of the $\Lambda_{b}^{0}$ and $\bar{B}^{0}$ hadrons. The relatively small value of $\left[M\left(\Lambda_{b}^{0}\right)-M\left(\Lambda_{c}^{+}\right)-M\left(D_{s}^{-}\right)\right]-$ $\left[M\left(\bar{B}^{0}\right)-M\left(D^{+}\right)-M\left(D_{s}^{-}\right)\right]$means that the uncertainty due to momentum scale, the dominant uncertainty in absolute-mass measurements, mostly cancels; however, it is still important to determine accurately the momenta of the final-state particles. The momentum-scale calibration of the spectrometer, which accounts for imperfect knowledge of the magnetic field and alignment, is discussed in detail in Refs. [12,34]. The uncertainty on the calibrated momentum scale is estimated to be $0.03 \%$ by comparing various particle masses measured at $\mathrm{LHCb}$ to their nominal values [34].

The kinematic and vertex constraints used in the fits described previously reduce the statistical uncertainty on $M\left(\Lambda_{b}^{0}\right)-M\left(\bar{B}^{0}\right)$ by improving the resolution. These

TABLE I. Relative systematic uncertainties on branching fraction measurements (\%). The production ratio $\sigma\left(B_{s}^{0}\right) / \sigma\left(\bar{B}^{0}\right)$ is taken from Ref. [31]. The numbers in parentheses in the last column are for the $B_{s}^{0}$ decay mode.

\begin{tabular}{lcccc}
\hline \hline Source & $\mathcal{B}\left(\Lambda_{b}^{0} \rightarrow \Lambda_{c}^{+} D^{-}\right) /$ & $\left(\left[\mathcal{B}\left(\Lambda_{b}^{0} \rightarrow \Lambda_{c}^{+} D_{s}^{-}\right) / \mathcal{B}\left(\bar{B}^{0} \rightarrow D^{+} D_{s}^{-}\right)\right]\right) /$ & $\mathcal{B}\left(B_{s}^{0} \rightarrow D^{+} D_{s}^{-}\right) /$ & $\mathcal{B}\left(B_{(s)}^{0} \rightarrow \Lambda_{c}^{+} \Lambda_{c}^{-}\right) /$ \\
& $\mathcal{B}\left(\Lambda_{b}^{0} \rightarrow \Lambda_{c}^{+} D_{s}^{-}\right)$ & $\left(\left[\mathcal{B}\left(\Lambda_{b}^{0} \rightarrow \Lambda_{c}^{+} \pi^{-}\right) / \mathcal{B}\left(\bar{B}^{0} \rightarrow D^{+} \pi^{-}\right)\right]\right)$ & $\mathcal{B}\left(\bar{B}^{0} \rightarrow D^{+} D_{s}^{-}\right)$ & $\mathcal{B}\left(B_{(s)}^{0} \rightarrow D^{+} D_{s}^{-}\right)$ \\
\hline Efficiency & 3.5 & 5.2 & 1.0 & $3.9(5.0)$ \\
Fit model & 3.0 & 2.6 & 3.0 & $\ldots$ \\
$\mathcal{B}\left(D_{(s)}^{+}, \Lambda_{c}^{+}\right)$ & 5.2 & $\cdots$ & $\ldots$ & 8.8 \\
$\sigma\left(B_{s}^{0}\right) / \sigma\left(\bar{B}^{0}\right)$ & $\ldots$ & $\ldots$ & 5.8 & $\ldots$ \\
$\sigma\left(\Lambda_{b}^{0}\right) / \sigma\left(\bar{B}^{0}\right)$ & $\ldots$ & 2.0 & $\ldots$ & $\ldots .6$ \\
Total & 6.9 & 6.1 & 6.6 & $9.1)$ \\
\hline \hline
\end{tabular}



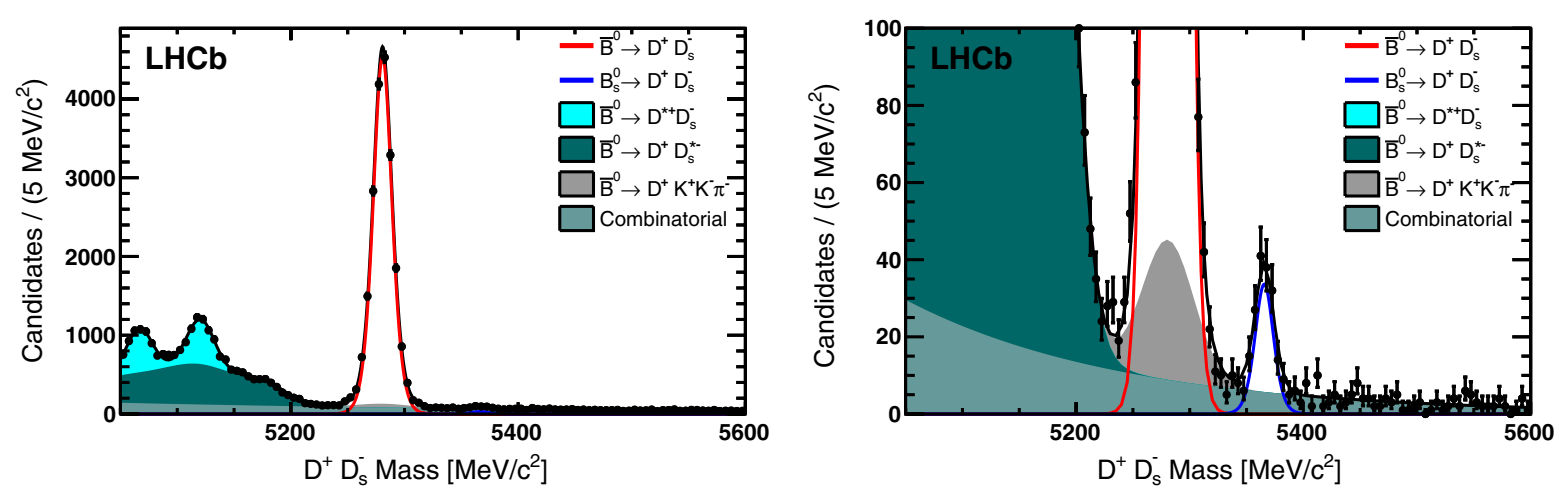

FIG. 2 (color online). Invariant mass distributions for $D^{+} D_{s}^{-}$candidates selected using BDT criteria optimized for the (left) $\bar{B}^{0} \rightarrow D^{+} D_{s}^{-}$and (right) $B_{s}^{0} \rightarrow D^{+} D_{s}^{-}$decay modes with the fits described in the text overlaid.

constraints also increase the systematic uncertainty by introducing a dependence on the precision of the nominal charm-hadron masses. These constraints are not imposed in the mass measurement, as it is found that this approach produces a smaller total uncertainty. The mass difference obtained is

$$
\begin{aligned}
M\left(\Lambda_{b}^{0}\right)-M\left(\bar{B}^{0}\right)= & 339.72 \pm 0.24(\text { stat }) \\
& \pm 0.18(\text { syst }) \mathrm{MeV} / c^{2} .
\end{aligned}
$$

The dominant systematic uncertainty (see Table II) arises due to a correlation between the reconstructed beautyhadron mass and reconstructed charm-hadron flight distance. The large difference in the $\Lambda_{c}^{+}$and $D^{+}$hadron lifetimes [2] could lead to only a partial cancellation of the biases induced by the charm-lifetime selection criteria. This effect is studied in simulation and a $0.16 \mathrm{MeV} / c^{2}$ uncertainty is assigned. The $0.03 \%$ uncertainty in the momentum scale results in an uncertainty on the mass difference of $0.08 \mathrm{MeV} / c^{2}$. Many variations in the fit model are considered, and none produce a significant shift in the mass

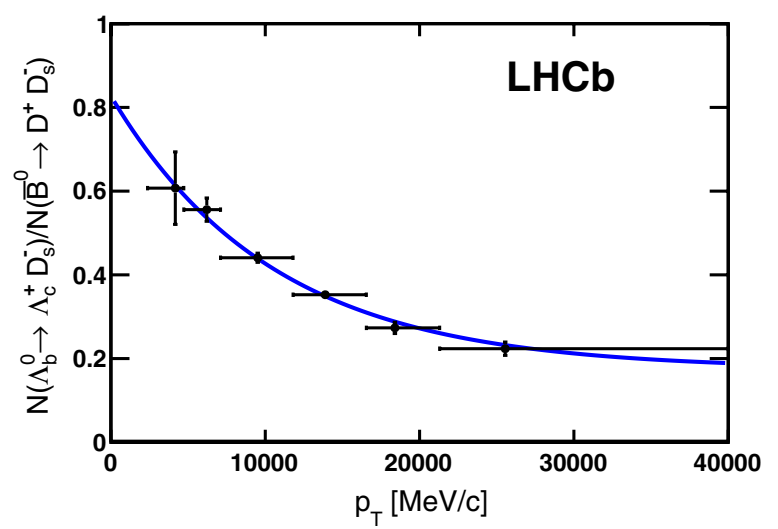

FIG. 3 (color online). Efficiency-corrected ratio of the yields of $\Lambda_{b}^{0} \rightarrow \Lambda_{c}^{+} D_{s}^{-}$and $\bar{B}^{0} \rightarrow D^{+} D_{s}^{-}$vs $p_{T}$. The points are located at the mean $p_{T}$ value of the $\Lambda_{b}^{0}$ in each bin. The curve shows the data fit with the shape of the $p_{T}$ dependence measured in Ref. [33]. difference. The systematic uncertainty in the mass difference due to the uncertainty in the amount of detector material in which charged particles lose energy is negligible [34]. Furthermore, the uncertainty on $M\left(\Lambda_{b}^{0}\right)-M\left(\bar{B}^{0}\right)$ due to differences in beauty-hadron production kinematics, as seen in Fig. 3, is also found to be negligible.

Using the nominal value for $M\left(\bar{B}^{0}\right)$ [2] gives $M\left(\Lambda_{b}^{0}\right)=$ $5619.30 \pm 0.34 \mathrm{MeV} / c^{2}$, where the uncertainty includes both statistical and systematic contributions. This is the most precise result to date. The total uncertainty is dominated by statistics and charm-hadron lifetime effects; thus, this result can be treated as being uncorrelated with the previous LHCb result obtained using the $\Lambda_{b}^{0} \rightarrow J / \psi \Lambda^{0}$ decay [35]. A weighted average of the $\mathrm{LHCb}$ results gives $M\left(\Lambda_{b}^{0}\right)=5619.36 \pm 0.26 \mathrm{MeV} / c^{2}$. This value may then be used to improve the precision of the $\Xi_{b}^{-}$and $\Omega_{b}^{-}$baryon masses using their mass differences with respect to the $\Lambda_{b}^{0}$ baryon, as reported in Ref. [35].

Using BDT criteria optimized for maximizing the expected significance of $B_{s}^{0} \rightarrow D^{+} D_{s}^{-}, 14608 \pm 121 \bar{B}^{0}$ and $143 \pm 14 B_{s}^{0}$ decays are observed (see Fig. 2), from which the ratio extracted is

$$
\frac{\mathcal{B}\left(B_{s}^{0} \rightarrow D^{+} D_{s}^{-}\right)}{\mathcal{B}\left(\bar{B}^{0} \rightarrow D^{+} D_{s}^{-}\right)}=0.038 \pm 0.004 \text { (stat) } \pm 0.003 \text { (syst) } .
$$

This is the most precise measurement to date of $\mathcal{B}\left(B_{s}^{0} \rightarrow D^{+} D_{s}^{-}\right)$and supersedes Ref. [36]. Since the two decay modes share the same final state, many systematic uncertainties cancel. The dominant contribution to the uncertainty comes from the beauty-hadron production fractions.

TABLE II. Systematic uncertainties for $M\left(\Lambda_{b}^{0}\right)-M\left(\bar{B}^{0}\right)$.

\begin{tabular}{lc}
\hline \hline Description & Value $\left(\mathrm{MeV} / c^{2}\right)$ \\
\hline$\Lambda_{c}^{+}-D^{+}$lifetime difference & 0.16 \\
Momentum scale & 0.08 \\
Fit model & 0.02 \\
Total & 0.18 \\
\hline \hline
\end{tabular}


A small additional uncertainty on the efficiency arises due to the uncertainty on the $B_{s}^{0}$ lifetime. Uncertainty in the fit model is largely due to the size of the combinatorial background near the $B_{s}^{0}$ peak. The measured ratio of branching fractions is approximately the ratio of quark-mixing factors, as expected assuming nonfactorizable effects are small.

A search is also performed for the decay modes $B_{(s)}^{0} \rightarrow \Lambda_{c}^{+} \Lambda_{c}^{-}$. Regions centered around the nominal $B_{(s)}^{0}$ meson masses with boundaries defined such that each region contains $95 \%$ of the corresponding signal are determined using simulation. The expected background contribution in each of these regions is obtained from the charm-hadron mass sidebands. Applying this technique to the $\bar{B}^{0} \rightarrow D^{+} D_{s}^{-}$and $\Lambda_{b}^{0} \rightarrow \Lambda_{c}^{+} D_{(s)}^{-}$decays produces background estimates consistent with those obtained by fitting the invariant mass spectra for those modes. The number of observed candidates in each signal region is then compared to the expected background contribution; no significant excess is observed in either $\Lambda_{c}^{+} \Lambda_{c}^{-}$signal region. The limits obtained using the method of Ref. [37] and the known $D_{s}^{-}$ [2], $D^{-}$[30], and $\Lambda_{c}^{+}$[38] hadron branching fractions are

$$
\begin{aligned}
& \frac{\mathcal{B}\left(\bar{B}^{0} \rightarrow \Lambda_{c}^{+} \Lambda_{c}^{-}\right)}{\mathcal{B}\left(\bar{B}^{0} \rightarrow D^{+} D_{s}^{-}\right)}<0.0022[95 \% \text { C.L. }], \\
& \frac{\mathcal{B}\left(B_{s}^{0} \rightarrow \Lambda_{c}^{+} \Lambda_{c}^{-}\right)}{\mathcal{B}\left(B_{s}^{0} \rightarrow D^{+} D_{s}^{-}\right)}<0.30[95 \% \text { C.L. }] .
\end{aligned}
$$

For these results the lifetime of the light-mass $B_{s}^{0}$ eigenstate is assumed, as this produces the most conservative limits [1]. This is the best limit to date for the $\bar{B}^{0}$ decay mode and the first limit for the $B_{s}^{0}$ decay mode.

In summary, first observations and relative branchingfraction measurements have been made for the decays $\Lambda_{b}^{0} \rightarrow \Lambda_{c}^{+} D_{(s)}^{-}$. The most precise measurements of the $\Lambda_{b}^{0}$ baryon mass and of $\mathcal{B}\left(B_{s}^{0} \rightarrow D^{+} D_{s}^{-}\right)$have been presented and the most stringent upper limits have been placed on $\mathcal{B}\left(B_{(s)}^{0} \rightarrow \Lambda_{c}^{+} \Lambda_{c}^{-}\right)$. Using $\mathcal{B}\left(\bar{B}^{0} \rightarrow D^{+} D_{s}^{-}\right)=(7.2 \pm 0.8) \times$ $10^{-3}{ }^{(s)}$ and $\mathcal{B}\left(\Lambda_{b}^{0} \rightarrow \Lambda_{c}^{+} \pi^{-}\right) / \mathcal{B}\left(\bar{B}^{0} \rightarrow D^{+} \pi^{-}\right)$from Ref. [33], the absolute branching fractions obtained are

$$
\begin{aligned}
\mathcal{B}\left(\Lambda_{b}^{0} \rightarrow \Lambda_{c}^{+} D_{s}^{-}\right) & =(1.1 \pm 0.1) \times 10^{-2}, \\
\mathcal{B}\left(\Lambda_{b}^{0} \rightarrow \Lambda_{c}^{+} D^{-}\right) & =(4.7 \pm 0.6) \times 10^{-4}, \\
\mathcal{B}\left(B_{s}^{0} \rightarrow D^{+} D_{s}^{-}\right) & =(2.7 \pm 0.5) \times 10^{-4}, \\
\mathcal{B}\left(\bar{B}^{0} \rightarrow \Lambda_{c}^{+} \Lambda_{c}^{-}\right) & <1.6 \times 10^{-5}[95 \% \text { C.L. }], \\
\mathcal{B}\left(B_{s}^{0} \rightarrow \Lambda_{c}^{+} \Lambda_{c}^{-}\right) & <8.0 \times 10^{-5}[95 \% \text { C.L. }] .
\end{aligned}
$$

These results are all consistent with expectations that assume small nonfactorizable effects.

We express our gratitude to our colleagues in the CERN accelerator departments for the excellent performance of the LHC. We thank the technical and administrative staff at the LHCb institutes. We acknowledge support from CERN and from the national agencies: CAPES, CNPq, FAPERJ, and FINEP (Brazil); NSFC (China); CNRS/IN2P3 and Region Auvergne (France); BMBF, DFG, HGF, and MPG (Germany); SFI (Ireland); INFN (Italy); FOM and NWO (The Netherlands); SCSR (Poland); MEN/IFA (Romania); MinES, Rosatom, RFBR, and NRC "Kurchatov Institute" (Russia); MinECo, XuntaGal, and GENCAT (Spain); SNSF and SER (Switzerland); NAS Ukraine (Ukraine); STFC (United Kingdom); NSF (USA). We also acknowledge the support received from EPLANET and the ERC under FP7. The Tier1 computing centers are supported by IN2P3 (France), KIT and BMBF (Germany), INFN (Italy), NWO and SURF (The Netherlands), PIC (Spain), GridPP (United Kingdom). We are indebted to the communities behind the multiple open-source software packages we depend on. We are also thankful for the computing resources and the access to software $R \& D$ tools provided by Yandex LLC (Russia).

[1] Y. Amhis et al. (Heavy Flavor Averaging Group), arXiv:1207.1158; updated results and plots available at http://www.slac.stanford.edu/xorg/hfag/.

[2] J. Beringer et al. (Particle Data Group), Phys. Rev. D 86, 010001 (2012), and 2013 partial update for the 2014 edition.

[3] M. Bauer, B. Stech, M. Wirbel, Z. Phys. C 34, 103 (1987).

[4] C. Amsler, T. Degrand, and B. Krusche, Quark Model, in Ref. [2], p. 199.

[5] M. Karliner, B. Keren-Zur, H. J. Lipkin, and J. L. Rosner, Ann. Phys. (N.Y.) 324, 2 (2009).

[6] J. P. Day, W. Plessas, and K.-S. Choi, arXiv:1205.6918.

[7] X. Liu, H.-X. Chen, Y.-R. Liu, A. Hosaka, and S.-L. Zhu, Phys. Rev. D 77, 014031 (2008).

[8] E. E. Jenkins, Phys. Rev. D 77, 034012 (2008).

[9] R. Roncaglia, D. Lichtenberg, and E. Predazzi, Phys. Rev. D 52, 1722 (1995).

[10] N. Mathur, R. Lewis, and R. Woloshyn, Phys. Rev. D 66, 014502 (2002).

[11] D. Ebert, R. Faustov, and V. Galkin, Phys. Rev. D 72, 034026 (2005).

[12] R. Aaij et al. (LHCb Collaboration), Phys. Lett. B 708, 241 (2012).

[13] A. A. Alves, Jr. et al. (LHCb Collaboration), JINST 3, S08005 (2008).

[14] R. Arink et al., JINST 9, P01002 (2014).

[15] M. Adinolfi et al., Eur. Phys. J. C 73, 2431 (2013).

[16] R. Aaij et al., JINST 8, P04022 (2013).

[17] A. A. Alves, Jr. et al., JINST 8, P02022 (2013).

[18] V. V. Gligorov and M. Williams, JINST 8, P02013 (2013).

[19] T. Sjöstrand, S. Mrenna, and P. Skands, J. High Energy Phys. 05 (2006) 026; T. Sjöstrand, S. Mrenna, and P. Skands, Comput. Phys. Commun. 178, 852 (2008).

[20] I. Belyaev et al., in Nuclear Science Symposium Conference Record (NSS/MIC), Knoxville, 2010 (IEEE, New York, 2010), p. 1155.

[21] D. J. Lange, Nucl. Instrum. Methods Phys. Res., Sect. A 462, 152 (2001).

[22] P. Golonka and Z. Was, Eur. Phys. J. C 45, 97 (2006). 
[23] J. Allison et al. (GEANT4 Collaboration), IEEE Trans. Nucl. Sci. 53, 270 (2006); S. Agostinelli et al. (GEANT4 Collaboration), Nucl. Instrum. Methods Phys. Res., Sect. A 506, 250 (2003).

[24] M. Clemencic, G. Corti, S. Easo, C. R. Jones, S. Miglioranzi, M. Pappagallo, and P. Robbe, J. Phys. Conf. Ser. 331, 032023 (2011).

[25] W. D. Hulsbergen, Nucl. Instrum. Methods Phys. Res., Sect. A 552, 566 (2005).

[26] L. Breiman, J. H. Friedman, R. A. Olshen, and C. J. Stone, Classification and Regression Trees (Wadsworth, Belmont, CA, 1984).

[27] M. Pivk and F. R. Le Diberder, Nucl. Instrum. Methods Phys. Res., Sect. A 555, 356 (2005).

[28] D. M. Santos and F. Dupertuis, arXiv:1312.5000.

[29] K. S. Cranmer, Comput. Phys. Commun. 136, 198 (2001).
[30] G. Bonvicini et al. (CLEO Collaboration), Phys. Rev. D 89, 072002 (2014).

[31] LHCb Collaboration, Report No. LHCb-CONF-2013-011.

[32] R. Aaij et al. (LHCb Collaboration), Phys. Rev. D 85, 032008 (2012).

[33] R. Aaij et al. (LHCb Collaboration), "Measurement of the $p_{T}$ and $\eta$ Dependences of $\Lambda_{b}^{0}$ Production and the $\Lambda_{b}^{0} \rightarrow \Lambda_{c}^{+} \pi^{-}$Branching Fraction" (to be published).

[34] R. Aaij et al. (LHCb Collaboration), J. High Energy Phys. 06 (2013) 65.

[35] R. Aaij et al. (LHCb Collaboration), Phys. Rev. Lett. 110, 182001 (2013).

[36] R. Aaij et al. (LHCb Collaboration), Phys. Rev. D 87, 092007 (2013).

[37] W. A. Rolke, A. M. Lopez, and J. Conrad, Nucl. Instrum. Methods Phys. Res., Sect. A 551, 493 (2005).

[38] A. Zupanc et al. (Belle Collaboration), arXiv:1312.7826.

R. Aaij, ${ }^{41}$ A. Abba,${ }^{21, a}$ B. Adeva,${ }^{37}$ M. Adinolfi, ${ }^{46}$ A. Affolder, ${ }^{52}$ Z. Ajaltouni, ${ }^{5}$ J. Albrecht, ${ }^{9}$ F. Alessio, ${ }^{38}$ M. Alexander, ${ }^{51}$ S. Ali, ${ }^{41}$ G. Alkhazov,${ }^{30}$ P. Alvarez Cartelle, ${ }^{37}$ A. A. Alves Jr., ${ }^{25,38}$ S. Amato, ${ }^{2}$ S. Amerio, ${ }^{22}$ Y. Amhis, ${ }^{7}$ L. An, ${ }^{3}$ L. Anderlini, ${ }^{17, b}$ J. Anderson, ${ }^{40}$ R. Andreassen, ${ }^{57}$ M. Andreotti, ${ }^{16, c}$ J. E. Andrews, ${ }^{58}$ R. B. Appleby, ${ }^{54}$ O. Aquines Gutierrez, ${ }^{10}$ F. Archilli, ${ }^{38}$ A. Artamonov, ${ }^{35}$ M. Artuso, ${ }^{59}$ E. Aslanides, ${ }^{6}$ G. Auriemma, ${ }^{25, d}$ M. Baalouch, ${ }^{5}$ S. Bachmann, ${ }^{11}$ J. J. Back, ${ }^{48}$ A. Badalov, ${ }^{36}$ V. Balagura, ${ }^{31}$ W. Baldini, ${ }^{16}$ R. J. Barlow, ${ }^{54}$ C. Barschel,${ }^{38}$ S. Barsuk, ${ }^{7}$ W. Barter, ${ }^{47}$ V. Batozskaya, ${ }^{28}$ T. Bauer, ${ }^{41}$ A. Bay ${ }^{39}$ J. Beddow, ${ }^{51}$ F. Bedeschi, ${ }^{23}$ I. Bediaga, ${ }^{1}$ S. Belogurov ${ }^{31}$ K. Belous,${ }^{35}$ I. Belyaev, ${ }^{31}$ E. Ben-Haim, ${ }^{8}$ G. Bencivenni, ${ }^{18}$ S. Benson, ${ }^{50}$ J. Benton, ${ }^{46}$ A. Berezhnoy, ${ }^{32}$ R. Bernet, ${ }^{40}$ M.-O. Bettler, ${ }^{47}$ M. van Beuzekom, ${ }^{41}$ A. Bien, ${ }^{11}$ S. Bifani, ${ }^{45}$ T. Bird, ${ }^{54}$ A. Bizzeti, ${ }^{17, e}$ P. M. Bjørnstad, ${ }^{54}$ T. Blake, ${ }^{48}$ F. Blanc, ${ }^{39}$ J. Blouw, ${ }^{10}$ S. Blusk, ${ }^{59}$ V. Bocci, ${ }^{25}$ A. Bondar, ${ }^{34}$ N. Bondar, ${ }^{30,38}$ W. Bonivento, ${ }^{15,38}$ S. Borghi, ${ }^{54}$ A. Borgia, ${ }^{59}$ M. Borsato, ${ }^{7}$ T. J. V. Bowcock, ${ }^{52}$ E. Bowen, ${ }^{40}$ C. Bozzi, ${ }^{16}$ T. Brambach, ${ }^{9}$ J. van den Brand, ${ }^{42}$ J. Bressieux, ${ }^{39}$ D. Brett, ${ }^{54}$ M. Britsch, ${ }^{10}$ T. Britton, ${ }^{59}$

N. H. Brook, ${ }^{46}$ H. Brown, ${ }^{52}$ A. Bursche, ${ }^{40}$ G. Busetto, ${ }^{22, f}$ J. Buytaert, ${ }^{38}$ S. Cadeddu, ${ }^{15}$ R. Calabrese, ${ }^{16, c}$ O. Callot, ${ }^{7}$ M. Calvi, ${ }^{20, g}$ M. Calvo Gomez, ${ }^{36, \mathrm{~h}}$ A. Camboni, ${ }^{36}$ P. Campana, ${ }^{18,38}$ D. Campora Perez, ${ }^{38}$ F. Caponio, ${ }^{21, \mathrm{a}}$ A. Carbone, ${ }^{14, \mathrm{i}}$ G. Carboni, ${ }^{24, j}$ R. Cardinale, ${ }^{19,38, \mathrm{k}}$ A. Cardini, ${ }^{15}$ H. Carranza-Mejia, ${ }^{50}$ L. Carson, ${ }^{50}$ K. Carvalho Akiba, ${ }^{2}$ G. Casse, ${ }^{52}$ L. Cassina, ${ }^{20}$ L. Castillo Garcia, ${ }^{38}$ M. Cattaneo,${ }^{38}$ C. Cauet, ${ }^{9}$ R. Cenci, ${ }^{58}$ M. Charles, ${ }^{8}$ P. Charpentier, ${ }^{38}$ S.-F. Cheung, ${ }^{55}$ N. Chiapolini, ${ }^{40}$ M. Chrzaszcz, ${ }^{40,26}$ K. Ciba,${ }^{38}$ X. Cid Vidal,${ }^{38}$ G. Ciezarek, ${ }^{53}$ P. E. L. Clarke, ${ }^{50}$ M. Clemencic,${ }^{38}$ H. V. Cliff, ${ }^{47}$ J. Closier, ${ }^{38}$ C. Coca, ${ }^{29}$ V. Coco, ${ }^{38}$ J. Cogan, ${ }^{6}$ E. Cogneras, ${ }^{5}$ P. Collins, ${ }^{38}$ A. Comerma-Montells, ${ }^{36}$ A. Contu, ${ }^{15,38}$ A. Cook, ${ }^{46}$ M. Coombes, ${ }^{46}$ S. Coquereau, ${ }^{8}$ G. Corti, ${ }^{38}$ M. Corvo, ${ }^{16, c}$ I. Counts, ${ }^{56}$ B. Couturier, ${ }^{38}$ G. A. Cowan, ${ }^{50}$ D. C. Craik, ${ }^{48}$ M. Cruz Torres ${ }^{60}$ A. R. Cukierman, ${ }^{56}$ S. Cunliffe, ${ }^{53}$ R. Currie, ${ }^{50}$ C. D'Ambrosio, ${ }^{38}$ J. Dalseno, ${ }^{46}$ P. David, ${ }^{8}$ P. N. Y. David, ${ }^{41}$ A. Davis,${ }^{57}$ K. De Bruyn, ${ }^{41}$ S. De Capua,${ }^{54}$ M. De Cian, ${ }^{11}$ J. M. De Miranda, ${ }^{1}$ L. De Paula, ${ }^{2}$ W. De Silva,${ }^{57}$ P. De Simone,${ }^{18}$ D. Decamp, ${ }^{4}$ M. Deckenhoff, ${ }^{9}$ L. Del Buono,${ }^{8}$ N. Déléage, ${ }^{4}$ D. Derkach, ${ }^{55}$ O. Deschamps, ${ }^{5}$ F. Dettori,${ }^{42}$ A. Di Canto ${ }^{38}$ H. Dijkstra, ${ }^{38}$ S. Donleavy, ${ }^{52}$ F. Dordei, ${ }^{11}$ M. Dorigo, ${ }^{39}$ C. Dorothy,${ }^{56}$ A. Dosil Suárez ${ }^{37}$ D. Dossett, ${ }^{48}$ A. Dovbnya ${ }^{43}$ F. Dupertuis, ${ }^{39}$ P. Durante, ${ }^{38}$ R. Dzhelyadin, ${ }^{35}$ A. Dziurda ${ }^{26}$ A. Dzyuba, ${ }^{30}$ S. Easo, ${ }^{49}$ U. Egede ${ }^{53}$ V. Egorychev,${ }^{31}$ S. Eidelman, ${ }^{34}$ S. Eisenhardt, ${ }^{50}$ U. Eitschberger, ${ }^{9}$ R. Ekelhof, ${ }^{9}$ L. Eklund,${ }^{51,38}$ I. El Rifai, ${ }^{5}$ C. Elsasser, ${ }^{40}$ S. Esen, ${ }^{11}$ T. Evans, ${ }^{55}$ A. Falabella, ${ }^{16, c}$ C. Färber, ${ }^{11}$ C. Farinelli, ${ }^{41}$ S. Farry, ${ }^{52}$ D. Ferguson, ${ }^{50}$ V. Fernandez Albor, ${ }^{37}$ F. Ferreira Rodrigues, ${ }^{1}$ M. Ferro-Luzzi ${ }^{38}$ S. Filippov ${ }^{33}$ M. Fiore, ${ }^{16, c}$ M. Fiorini, ${ }^{16, c}$ M. Firlej, ${ }^{27}$ C. Fitzpatrick, ${ }^{38}$ T. Fiutowski, ${ }^{27}$ M. Fontana, ${ }^{10}$ F. Fontanelli,,${ }^{19, k}$ R. Forty ${ }^{38}$ O. Francisco, ${ }^{2}$ M. Frank,${ }^{38}$ C. Frei,${ }^{38}$ M. Frosini, ${ }^{17,38, b}$ J. Fu, ${ }^{21}$ E. Furfaro, ${ }^{24, j}$ A. Gallas Torreira, ${ }^{37}$ D. Galli, ${ }^{14, \mathrm{i}}$ S. Gambetta, ${ }^{19, k}$ M. Gandelman, ${ }^{2}$ P. Gandini, ${ }^{59}$ Y. Gao, ${ }^{3}$ J. Garofoli, ${ }^{59}$ J. Garra Tico, ${ }^{47}$ L. Garrido, ${ }^{36}$ C. Gaspar, ${ }^{38}$ R. Gauld, ${ }^{55}$ L. Gavardi, ${ }^{9}$ E. Gersabeck, ${ }^{11}$ M. Gersabeck, ${ }^{54}$ T. Gershon, ${ }^{48}$ P. Ghez, ${ }^{4}$ A. Gianelle, ${ }^{22}$ S. Giani, ${ }^{39}$ V. Gibson, ${ }^{47}$ L. Giubega, ${ }^{29}$ V. V. Gligorov, ${ }^{38}$ C. Göbel, ${ }^{60}$ D. Golubkov, ${ }^{31}$ A. Golutvin, ${ }^{53,31,38}$ A. Gomes, ${ }^{1,1}$ H. Gordon, ${ }^{38}$ C. Gotti, ${ }^{20}$ M. Grabalosa Gándara, ${ }^{5}$ R. Graciani Diaz, ${ }^{36}$ L. A. Granado Cardoso, ${ }^{38}$ E. Graugés,${ }^{36}$ G. Graziani, ${ }^{17}$ A. Grecu, ${ }^{29}$ E. Greening, ${ }^{55}$ S. Gregson, ${ }^{47}$ P. Griffith ${ }^{45}$ L. Grillo, ${ }^{11}$ O. Grünberg, ${ }^{62}$ B. Gui, ${ }^{59}$ E. Gushchin, ${ }^{33}$ Y. Guz,${ }^{35,38}$ T. Gys, ${ }^{38}$ C. Hadjivasiliou, ${ }^{59}$ G. Haefeli, ${ }^{39}$ C. Haen, ${ }^{38}$ S. C. Haines, ${ }^{47}$ S. Hall,${ }^{53}$ B. Hamilton, ${ }^{58}$ T. Hampson, ${ }^{46}$ X. Han, ${ }^{11}$ 
S. Hansmann-Menzemer, ${ }^{11}$ N. Harnew,${ }^{55}$ S. T. Harnew, ${ }^{46}$ J. Harrison,${ }^{54}$ T. Hartmann,${ }^{62}$ J. He, ${ }^{38}$ T. Head, ${ }^{38}$ V. Heijne, ${ }^{41}$ K. Hennessy, ${ }^{52}$ P. Henrard, ${ }^{5}$ L. Henry, ${ }^{8}$ J. A. Hernando Morata, ${ }^{37}$ E. van Herwijnen, ${ }^{38}$ M. Heß,${ }^{62}$ A. Hicheur, ${ }^{1}$ D. Hill, ${ }^{55}$ M. Hoballah, ${ }^{5}$ C. Hombach, ${ }^{54}$ W. Hulsbergen, ${ }^{41}$ P. Hunt,${ }^{55}$ N. Hussain, ${ }^{55}$ D. Hutchcroft, ${ }^{52}$ D. Hynds,${ }^{51}$ M. Idzik, ${ }^{27}$ P. Ilten, ${ }^{56}$ R. Jacobsson, ${ }^{38}$ A. Jaeger, ${ }^{11}$ J. Jalocha, ${ }^{55}$ E. Jans, ${ }^{41}$ P. Jaton, ${ }^{39}$ A. Jawahery,${ }^{58}$ M. Jezabek, ${ }^{26}$ F. Jing, ${ }^{3}$ M. John, ${ }^{55}$ D. Johnson, ${ }^{55}$ C. R. Jones, ${ }^{47}$ C. Joram, ${ }^{38}$ B. Jost,${ }^{38}$ N. Jurik, ${ }^{59}$ M. Kaballo, ${ }^{9}$ S. Kandybei,${ }^{43}$ W. Kanso, ${ }^{6}$ M. Karacson, ${ }^{38}$ T. M. Karbach, ${ }^{38}$ M. Kelsey, ${ }^{59}$ I. R. Kenyon, ${ }^{45}$ T. Ketel, ${ }^{42}$ B. Khanji, ${ }^{20}$ C. Khurewathanakul, ${ }^{39}$ S. Klaver, ${ }^{54}$ O. Kochebina, ${ }^{7}$ M. Kolpin, ${ }^{11}$ I. Komarov, ${ }^{39}$ R. F. Koopman, ${ }^{42}$ P. Koppenburg, ${ }^{41,38}$ M. Korolev, ${ }^{32}$ A. Kozlinskiy, ${ }^{41}$ L. Kravchuk, ${ }^{33}$ K. Kreplin, ${ }^{11}$ M. Kreps, ${ }^{48}$ G. Krocker, ${ }^{11}$ P. Krokovny, ${ }^{34}$ F. Kruse, ${ }^{9}$ M. Kucharczyk, ${ }^{20,26,38, g}$ V. Kudryavtsev, ${ }^{34}$ K. Kurek,${ }^{28}$ T. Kvaratskheliya, ${ }^{31}$ V. N. La Thi, ${ }^{39}$ D. Lacarrere, ${ }^{38}$ G. Lafferty, ${ }^{54}$ A. Lai, ${ }^{15}$ D. Lambert, ${ }^{50}$ R. W. Lambert, ${ }^{42}$ E. Lanciotti, ${ }^{38}$ G. Lanfranchi, ${ }^{18}$ C. Langenbruch, ${ }^{38}$ B. Langhans, ${ }^{38}$ T. Latham, ${ }^{48}$ C. Lazzeroni, ${ }^{45}$ R. Le Gac, ${ }^{6}$ J. van Leerdam,${ }^{41}$ J.-P. Lees, ${ }^{4}$ R. Lefèvre, ${ }^{5}$ A. Leflat, ${ }^{32}$ J. Lefrançois, ${ }^{7}$ S. Leo, ${ }^{23}$ O. Leroy, ${ }^{6}$ T. Lesiak, ${ }^{26}$ B. Leverington, ${ }^{11}$ Y. Li,${ }^{3}$ M. Liles, ${ }^{52}$ R. Lindner,${ }^{38}$ C. Linn,${ }^{38}$ F. Lionetto, ${ }^{40}$ B. Liu, ${ }^{15}$ G. Liu, ${ }^{38}$ S. Lohn, ${ }^{38}$ I. Longstaff, ${ }^{51}$ I. Longstaff, ${ }^{51}$ J. H. Lopes, ${ }^{2}$ N. Lopez-March, ${ }^{39}$ P. Lowdon, ${ }^{40}$ H. Lu, ${ }^{3}$ D. Lucchesi,${ }^{22, f}$ H. Luo, ${ }^{50}$ A. Lupato, ${ }^{22}$ E. Luppi, ${ }^{16, c}$ O. Lupton,${ }^{55}$ F. Machefert, ${ }^{7}$ I. V. Machikhiliyan, ${ }^{31}$ F. Maciuc, ${ }^{29}$ O. Maev, ${ }^{30}$ S. Malde,${ }^{55}$ G. Manca, ${ }^{15, \mathrm{~m}}$ G. Mancinelli ${ }^{6}$ M. Manzali, ${ }^{16, \mathrm{c}}$ J. Maratas, ${ }^{5}$ J. F. Marchand, ${ }^{4}$ U. Marconi, ${ }^{14}$ C. Marin Benito, ${ }^{36}$ P. Marino, ${ }^{23, n}$ R. Märki, ${ }^{39}$ J. Marks, ${ }^{11}$ G. Martellotti, ${ }^{25}$ A. Martens, ${ }^{8}$ A. Martín Sánchez, M. Martinelli, ${ }^{41}$ D. Martinez Santos, ${ }^{42}$ F. Martinez Vidal, ${ }^{64}$ D. Martins Tostes, ${ }^{2}$ A. Massafferri, ${ }^{1}$ R. Matev ${ }^{38}$ Z. Mathe, ${ }^{38}$ C. Matteuzzi, ${ }^{20}$ A. Mazurov, ${ }^{16,38, c}$ M. McCann,${ }^{53}$ J. McCarthy, ${ }^{45}$ A. McNab,${ }^{54}$ R. McNulty, ${ }^{12}$ B. McSkelly,${ }^{52}$ B. Meadows, ${ }^{57,55}$ F. Meier, ${ }^{9}$ M. Meissner, ${ }^{11}$ M. Merk, ${ }^{41}$ D. A. Milanes, ${ }^{8}$ M.-N. Minard, ${ }^{4}$ J. Molina Rodriguez,${ }^{60}$ S. Monteil,${ }^{5}$ D. Moran, ${ }^{54}$ M. Morandin, ${ }^{22}$ P. Morawski, ${ }^{26}$ A. Mordà, ${ }^{6}$ M. J. Morello, ${ }^{23, n}$ J. Moron, ${ }^{27}$ R. Mountain, ${ }^{59}$ F. Muheim, ${ }^{50}$ K. Müller, ${ }^{40}$ R. Muresan, ${ }^{29}$ B. Muster, ${ }^{39}$ P. Naik,${ }^{46}$ T. Nakada,${ }^{39}$ R. Nandakumar, ${ }^{49}$ I. Nasteva, ${ }^{1}$ M. Needham,${ }^{50}$ N. Neri, ${ }^{21}$ S. Neubert, ${ }^{38}$ N. Neufeld, ${ }^{38}$ M. Neuner, ${ }^{11}$ A. D. Nguyen, ${ }^{39}$ T. D. Nguyen,,${ }^{39}$ C. Nguyen-Mau,,${ }^{39,0}$ M. Nicol, ${ }^{7}$ V. Niess, ${ }^{5}$ R. Niet, ${ }^{9}$ N. Nikitin, ${ }^{32}$ T. Nikodem, ${ }^{11}$ A. Novoselov ${ }^{35}$ A. Oblakowska-Mucha, ${ }^{27}$ V. Obraztsov, ${ }^{35}$ S. Oggero, ${ }^{41}$ S. Ogilvy, ${ }^{51}$ O. Okhrimenko, ${ }^{44}$ R. Oldeman, ${ }^{15, \mathrm{~m}}$ G. Onderwater, ${ }^{65}$ M. Orlandea, ${ }^{29}$ J. M. Otalora Goicochea, ${ }^{2}$ P. Owen, ${ }^{53}$ A. Oyanguren, ${ }^{64}$ B. K. Pal,${ }^{59}$ A. Palano, ${ }^{13, \mathrm{p}}$ F. Palombo, ${ }^{21, \mathrm{q}}$ M. Palutan, ${ }^{18}$ J. Panman, ${ }^{38}$ A. Papanestis, ${ }^{49,38}$ M. Pappagallo, ${ }^{51}$ C. Parkes,${ }^{54}$ C. J. Parkinson, ${ }^{9}$ G. Passaleva, ${ }^{17}$ G. D. Patel,${ }^{52}$ M. Patel,${ }^{53}$ C. Patrignani, ${ }^{19, k}$ A. Pazos Alvarez, ${ }^{37}$ A. Pearce, ${ }^{54}$ A. Pellegrino, ${ }^{41}$ M. Pepe Altarelli, ${ }^{38}$ S. Perazzini, ${ }^{14, i}$ E. Perez Trigo, ${ }^{37}$ P. Perret, ${ }^{5}$ M. Perrin-Terrin, ${ }^{6}$ L. Pescatore, ${ }^{45}$ E. Pesen, ${ }^{66}$ K. Petridis,${ }^{53}$ A. Petrolini, ${ }^{19, k}$ E. Picatoste Olloqui, ${ }^{36}$ B. Pietrzyk, ${ }^{4}$ T. Pilař, ${ }^{48}$ D. Pinci, ${ }^{25}$ A. Pistone, ${ }^{19}$ S. Playfer, ${ }^{50}$ M. Plo Casasus,${ }^{37}$

F. Polci, ${ }^{8}$ A. Poluektov, ${ }^{48,34}$ E. Polycarpo, ${ }^{2}$ A. Popov, ${ }^{35}$ D. Popov, ${ }^{10}$ B. Popovici,${ }^{29}$ C. Potterat,${ }^{2}$ A. Powell, ${ }^{55}$ J. Prisciandaro, ${ }^{39}$ A. Pritchard ${ }^{52}$ C. Prouve, ${ }^{46}$ V. Pugatch, ${ }^{44}$ A. Puig Navarro, ${ }^{39}$ G. Punzi, ${ }^{23, r}$ W. Qian, ${ }^{4}$ B. Rachwal, ${ }^{26}$ J. H. Rademacker, ${ }^{46}$ B. Rakotomiaramanana, ${ }^{39}$ M. Rama, ${ }^{18}$ M. S. Rangel,${ }^{2}$ I. Raniuk, ${ }^{43}$ N. Rauschmayr,${ }^{38}$ G. Raven, ${ }^{42}$ S. Reichert, ${ }^{54}$ M. M. Reid, ${ }^{48}$ A. C. dos Reis, ${ }^{1}$ S. Ricciardi, ${ }^{49}$ A. Richards,${ }^{53}$ K. Rinnert, ${ }^{52}$ V. Rives Molina,${ }^{36}$ D. A. Roa Romero, ${ }^{5}$ P. Robbe, ${ }^{7}$ A. B. Rodrigues, ${ }^{1}$ E. Rodrigues,${ }^{54}$ P. Rodriguez Perez,${ }^{54}$ S. Roiser, ${ }^{38}$ V. Romanovsky, ${ }^{35}$ A. Romero Vidal, ${ }^{37}$ M. Rotondo, ${ }^{22}$ J. Rouvinet, ${ }^{39}$ T. Ruf, ${ }^{38}$ F. Ruffini, ${ }^{23}$ H. Ruiz, ${ }^{36}$ P. Ruiz Valls, ${ }^{64}$ G. Sabatino, ${ }^{25, j}$ J. J. Saborido Silva, ${ }^{37}$

N. Sagidova ${ }^{30}$ P. Sail,${ }^{51}$ B. Saitta, ${ }^{15, \mathrm{~m}}$ V. Salustino Guimaraes, ${ }^{2}$ C. Sanchez Mayordomo, ${ }^{64}$ B. Sanmartin Sedes, ${ }^{37}$ R. Santacesaria, ${ }^{25}$ C. Santamarina Rios,${ }^{37}$ E. Santovetti, ${ }^{24, j}$ M. Sapunov, ${ }^{6}$ A. Sarti, ${ }^{18, \mathrm{~s}}$ C. Satriano, ${ }^{25, \mathrm{~d}}$ A. Satta,${ }^{24}$ M. Savrie, ${ }^{16,}$ ${ }^{\mathrm{c}}$ D. Savrina, ${ }^{31,32}$ M. Schiller, ${ }^{42}$ H. Schindler, ${ }^{38}$ M. Schlupp, ${ }^{9}$ M. Schmelling, ${ }^{10}$ B. Schmidt,${ }^{38}$ O. Schneider, ${ }^{39}$ A. Schopper, ${ }^{38}$ M.-H. Schune, ${ }^{7}$ R. Schwemmer, ${ }^{38}$ B. Sciascia, ${ }^{18}$ A. Sciubba,${ }^{25}$ M. Seco, ${ }^{37}$ A. Semennikov, ${ }^{31}$ K. Senderowska, ${ }^{27}$ I. Sepp,${ }^{53}$

N. Serra, ${ }^{40}$ J. Serrano, ${ }^{6}$ L. Sestini, ${ }^{22}$ P. Seyfert, ${ }^{11}$ M. Shapkin, ${ }^{35}$ I. Shapoval, ${ }^{16,43, \mathrm{c}}$ Y. Shcheglov, ${ }^{30}$ T. Shears, ${ }^{52}$ L. Shekhtman, ${ }^{34}$ V. Shevchenko, ${ }^{63}$ A. Shires, ${ }^{9}$ R. Silva Coutinho, ${ }^{48}$ G. Simi,${ }^{22}$ M. Sirendi, ${ }^{47}$ N. Skidmore,${ }^{46}$ T. Skwarnicki, ${ }^{59}$ N. A. Smith, ${ }^{52}$ E. Smith, ${ }^{55,49}$ E. Smith, ${ }^{53}$ J. Smith, ${ }^{47}$ M. Smith, ${ }^{54}$ H. Snoek, ${ }^{41}$ M. D. Sokoloff, ${ }^{57}$ F. J. P. Soler, ${ }^{51}$ F. Soomro, ${ }^{39}$ D. Souza, ${ }^{46}$ B. Souza De Paula, ${ }^{2}$ B. Spaan, ${ }^{9}$ A. Sparkes,${ }^{50}$ F. Spinella, ${ }^{23}$ P. Spradlin, ${ }^{51}$ F. Stagni, ${ }^{38}$ S. Stahl, ${ }^{11}$ O. Steinkamp, ${ }^{40}$ O. Stenyakin, ${ }^{35}$ S. Stevenson, ${ }^{55}$ S. Stoica, ${ }^{29}$ S. Stone, ${ }^{59}$ B. Storaci, ${ }^{40}$ S. Stracka, ${ }^{23,38}$ M. Straticiuc, ${ }^{29}$ U. Straumann, ${ }^{40}$ R. Stroili, ${ }^{22}$ V. K. Subbiah, ${ }^{38}$ L. Sun,${ }^{57}$ W. Sutcliffe, ${ }^{53}$ K. Swientek, ${ }^{27}$ S. Swientek, ${ }^{9}$ V. Syropoulos, ${ }^{42}$ M. Szczekowski, ${ }^{28}$ P. Szczypka, ${ }^{39,38}$ D. Szilard, ${ }^{2}$ T. Szumlak, ${ }^{27}$ S. T'Jampens, ${ }^{4}$ M. Teklishyn, ${ }^{7}$ G. Tellarini, ${ }^{16, c}$ E. Teodorescu, ${ }^{29}$ F. Teubert, ${ }^{38}$ C. Thomas, ${ }^{55}$ E. Thomas, ${ }^{38}$ J. van Tilburg, ${ }^{41}$ V. Tisserand, ${ }^{4}$ M. Tobin, ${ }^{39}$ S. Tolk,${ }^{42}$ L. Tomassetti,,${ }^{16, c}$ D. Tonelli, ${ }^{38}$ S. ToppJoergensen, ${ }^{55}$ N. Torr, ${ }^{55}$ E. Tournefier, ${ }^{4}$ S. Tourneur, ${ }^{39}$ M. T. Tran, ${ }^{39}$ M. Tresch, ${ }^{40}$ A. Tsaregorodtsev, ${ }^{6}$ P. Tsopelas, ${ }^{41}$ N. Tuning, ${ }^{41}$ M. Ubeda Garcia, ${ }^{38}$ A. Ukleja, ${ }^{28}$ A. Ustyuzhanin, ${ }^{63}$ U. Uwer, ${ }^{11}$ V. Vagnoni, ${ }^{14}$ G. Valenti, ${ }^{14}$ A. Vallier, ${ }^{7}$ R. Vazquez Gomez, ${ }^{18}$ P. Vazquez Regueiro, ${ }^{37}$ C. Vázquez Sierra, ${ }^{37}$ S. Vecchi,${ }^{16}$ J. J. Velthuis,${ }^{46}$ M. Veltri, ${ }^{17, t}$ G. Veneziano,${ }^{39}$ M. Vesterinen, ${ }^{11}$ B. Viaud, ${ }^{7}$ D. Vieira, ${ }^{2}$ M. Vieites Diaz, ${ }^{37}$ X. Vilasis-Cardona, ${ }^{36, h}$ A. Vollhardt, ${ }^{40}$ D. Volyanskyy, ${ }^{10}$ 
D. Voong, ${ }^{46}$ A. Vorobyev,${ }^{30}$ V. Vorobyev,${ }^{34}$ C. Voß,${ }^{62}$ H. Voss,${ }^{10}$ J. A. de Vries,${ }^{41}$ R. Waldi, ${ }^{62}$ C. Wallace, ${ }^{48}$ R. Wallace, ${ }^{12}$ J. Walsh, ${ }^{23}$ S. Wandernoth, ${ }^{11}$ J. Wang,${ }^{59}$ D. R. Ward, ${ }^{47}$ N. K. Watson, ${ }^{45}$ A. D. Webber,${ }^{54}$ D. Websdale,${ }^{53}$ M. Whitehead, ${ }^{48}$ J. Wicht ${ }^{38}$ D. Wiedner, ${ }^{11}$ G. Wilkinson, ${ }^{55}$ M. P. Williams, ${ }^{45}$ M. Williams, ${ }^{56}$ F. F. Wilson, ${ }^{49}$ J. Wimberley,${ }^{58}$ J. Wishahi, ${ }^{9}$ W. Wislicki, ${ }^{28}$ M. Witek, ${ }^{26}$ G. Wormser, ${ }^{7}$ S. A. Wotton, ${ }^{47}$ S. Wright,${ }^{47}$ S. Wu, ${ }^{3}$ K. Wyllie, ${ }^{38}$ Y. Xie, ${ }^{61}$ Z. Xing, ${ }^{59}$ Z. Xu, ${ }^{39}$ Z. Yang, ${ }^{3}$ X. Yuan, ${ }^{3}$ O. Yushchenko, ${ }^{35}$ M. Zangoli, ${ }^{14}$ M. Zavertyaev, ${ }^{10, u}$ F. Zhang, ${ }^{3}$ L. Zhang, ${ }^{59}$ W. C. Zhang, ${ }^{12}$ Y. Zhang, ${ }^{3}$ A. Zhelezov, ${ }^{11}$ A. Zhokhov, ${ }^{31}$ L. Zhong ${ }^{3}$ and A. Zvyagin ${ }^{38}$

\section{(LHCb Collaboration)}

${ }^{1}$ Centro Brasileiro de Pesquisas Físicas (CBPF), Rio de Janeiro, Brazil

${ }^{2}$ Universidade Federal do Rio de Janeiro (UFRJ), Rio de Janeiro, Brazil

${ }^{3}$ Center for High Energy Physics, Tsinghua University, Beijing, China

${ }^{4} L A P P$, Université de Savoie, CNRS/IN2P3, Annecy-Le-Vieux, France

${ }^{5}$ Clermont Université, Université Blaise Pascal, CNRS/IN2P3, LPC, Clermont-Ferrand, France

${ }^{6}$ CPPM, Aix-Marseille Université, CNRS/IN2P3, Marseille, France

${ }^{7} L A L$, Université Paris-Sud, CNRS/IN2P3, Orsay, France

${ }^{8}$ LPNHE, Université Pierre et Marie Curie, Université Paris Diderot, CNRS/IN2P3, Paris, France

${ }^{9}$ Fakultät Physik, Technische Universität Dortmund, Dortmund, Germany

${ }^{10}$ Max-Planck-Institut für Kernphysik (MPIK), Heidelberg, Germany

${ }^{11}$ Physikalisches Institut, Ruprecht-Karls-Universität Heidelberg, Heidelberg, Germany

${ }^{12}$ School of Physics, University College Dublin, Dublin, Ireland

${ }^{13}$ Sezione INFN di Bari, Bari, Italy

${ }^{14}$ Sezione INFN di Bologna, Bologna, Italy

${ }^{15}$ Sezione INFN di Cagliari, Cagliari, Italy

${ }^{16}$ Sezione INFN di Ferrara, Ferrara, Italy

${ }^{17}$ Sezione INFN di Firenze, Firenze, Italy

${ }^{18}$ Laboratori Nazionali dell'INFN di Frascati, Frascati, Italy

${ }^{19}$ Sezione INFN di Genova, Genova, Italy

${ }^{20}$ Sezione INFN di Milano Bicocca, Milano, Italy

${ }^{21}$ Sezione INFN di Milano, Milano, Italy

${ }^{22}$ Sezione INFN di Padova, Padova, Italy

${ }^{23}$ Sezione INFN di Pisa, Pisa, Italy

${ }^{24}$ Sezione INFN di Roma Tor Vergata, Roma, Italy

${ }^{25}$ Sezione INFN di Roma La Sapienza, Roma, Italy

${ }^{26}$ Henryk Niewodniczanski Institute of Nuclear Physics Polish Academy of Sciences, Kraków, Poland

${ }^{27}$ AGH - University of Science and Technology, Faculty of Physics and Applied Computer Science, Kraków, Poland

${ }^{28}$ National Center for Nuclear Research (NCBJ), Warsaw, Poland

${ }^{29}$ Horia Hulubei National Institute of Physics and Nuclear Engineering, Bucharest-Magurele, Romania

${ }^{30}$ Petersburg Nuclear Physics Institute (PNPI), Gatchina, Russia

${ }^{31}$ Institute of Theoretical and Experimental Physics (ITEP), Moscow, Russia

${ }^{32}$ Institute of Nuclear Physics, Moscow State University (SINP MSU), Moscow, Russia

${ }^{33}$ Institute for Nuclear Research of the Russian Academy of Sciences (INR RAN), Moscow, Russia

${ }^{34}$ Budker Institute of Nuclear Physics (SB RAS) and Novosibirsk State University, Novosibirsk, Russia

${ }^{35}$ Institute for High Energy Physics (IHEP), Protvino, Russia

${ }^{36}$ Universitat de Barcelona, Barcelona, Spain

${ }^{37}$ Universidad de Santiago de Compostela, Santiago de Compostela, Spain

${ }^{38}$ European Organization for Nuclear Research (CERN), Geneva, Switzerland

${ }^{39}$ Ecole Polytechnique Fédérale de Lausanne (EPFL), Lausanne, Switzerland

${ }^{40}$ Physik-Institut, Universität Zürich, Zürich, Switzerland

${ }^{41}$ Nikhef National Institute for Subatomic Physics, Amsterdam, The Netherlands

${ }^{42}$ Nikhef National Institute for Subatomic Physics and VU University Amsterdam, Amsterdam, The Netherlands

${ }^{43}$ NSC Kharkiv Institute of Physics and Technology (NSC KIPT), Kharkiv, Ukraine

${ }^{44}$ Institute for Nuclear Research of the National Academy of Sciences (KINR), Kyiv, Ukraine

${ }^{45}$ University of Birmingham, Birmingham, United Kingdom

${ }^{46}$ H.H. Wills Physics Laboratory, University of Bristol, Bristol, United Kingdom

${ }^{47}$ Cavendish Laboratory, University of Cambridge, Cambridge, United Kingdom

${ }^{48}$ Department of Physics, University of Warwick, Coventry, United Kingdom

${ }^{49}$ STFC Rutherford Appleton Laboratory, Didcot, United Kingdom 
${ }^{50}$ School of Physics and Astronomy, University of Edinburgh, Edinburgh, United Kingdom

${ }^{51}$ School of Physics and Astronomy, University of Glasgow, Glasgow, United Kingdom

${ }^{52}$ Oliver Lodge Laboratory, University of Liverpool, Liverpool, United Kingdom

${ }^{53}$ Imperial College London, London, United Kingdom

${ }^{54}$ School of Physics and Astronomy, University of Manchester, Manchester, United Kingdom

${ }^{55}$ Department of Physics, University of Oxford, Oxford, United Kingdom

${ }^{56}$ Massachusetts Institute of Technology, Cambridge, Massachusetts, USA

${ }^{57}$ University of Cincinnati, Cincinnati, Ohio, USA

${ }^{58}$ University of Maryland, College Park, Maryland, USA

${ }^{59}$ Syracuse University, Syracuse, New York, USA

${ }^{60}$ Pontifícia Universidade Católica do Rio de Janeiro (PUC-Rio), Rio de Janeiro, Brazil (associated with Universidade Federal do Rio de Janeiro (UFRJ), Rio de Janeiro, Brazil)

${ }^{61}$ Institute of Particle Physics, Central China Normal University, Wuhan, Hubei, China (associated with Center for High Energy Physics, Tsinghua University, Beijing, China)

${ }^{62}$ Institut für Physik, Universität Rostock, Rostock, Germany (associated with Physikalisches Institut, Ruprecht-Karls-Universität Heidelberg, Heidelberg, Germany)

${ }^{63}$ National Research Centre Kurchatov Institute, Moscow, Russia (associated with Institute of Theoretical and Experimental Physics [ITEP], Moscow, Russia)

${ }^{64}$ Instituto de Fisica Corpuscular (IFIC), Universitat de Valencia-CSIC, Valencia, Spain (associated with Universitat de Barcelona, Barcelona, Spain)

${ }^{65}$ KVI-University of Groningen, Groningen, The Netherlands (associated with Nikhef National Institute for Subatomic Physics, Amsterdam, The Netherlands)

${ }^{66}$ Celal Bayar University, Manisa, Turkey (associated with European Organization for Nuclear Research [CERN], Geneva, Switzerland)

${ }^{a}$ Also at Politecnico di Milano, Milano, Italy.

${ }^{\mathrm{b}}$ Also at Università di Firenze, Firenze, Italy.

${ }^{\mathrm{c}}$ Also at Università di Ferrara, Ferrara, Italy.

${ }^{\mathrm{d}}$ Also at Università della Basilicata, Potenza, Italy.

${ }^{\mathrm{e}}$ Also at Università di Modena e Reggio Emilia, Modena, Italy.

${ }^{\mathrm{f}}$ Also at Università di Padova, Padova, Italy.

${ }^{g}$ Also at Università di Milano Bicocca, Milano, Italy.

${ }^{\mathrm{h}}$ Also at LIFAELS, La Salle, Universitat Ramon Llull, Barcelona, Spain.

${ }^{\mathrm{i}}$ Also at Università di Bologna, Bologna, Italy.

${ }^{\mathrm{j}}$ Also at Università di Roma Tor Vergata, Roma, Italy.

${ }^{\mathrm{k}}$ Also at Università di Genova, Genova, Italy.

${ }^{1}$ Also at Universidade Federal do Triângulo Mineiro (UFTM), Uberaba-MG, Brazil.

${ }^{\mathrm{m}}$ Also at Università di Cagliari, Cagliari, Italy.

${ }^{\mathrm{n}}$ Also at Scuola Normale Superiore, Pisa, Italy.

${ }^{\circ}$ Also at Hanoi University of Science, Hanoi, Viet Nam.

${ }^{\mathrm{p}}$ Also at Università di Bari, Bari, Italy.

${ }^{\mathrm{q}}$ Also at Università degli Studi di Milano, Milano, Italy.

${ }^{\mathrm{r}}$ Also at Università di Pisa, Pisa, Italy.

s Also at Università di Roma La Sapienza, Roma, Italy.

${ }^{\mathrm{t}}$ Also at Università di Urbino, Urbino, Italy.

${ }^{\mathrm{u}}$ Also at P.N. Lebedev Physical Institute, Russian Academy of Science (LPI RAS), Moscow, Russia. 